\title{
The structure of nuclear systems derived from low momentum nucleon-nucleon potentials
}

\author{
J. Kuckei, F. Montani, H. Müther and A. Sedrakian \\ Institut für Theoretische Physik, Universität Tübingen, D-720\%6 Tübingen, \\ Germany
}

\begin{abstract}
Various nuclear structure observables are evaluated employing low-momentum nucleonnucleon (NN) potentials $V_{\text {low-k }}$ derived from the CD-Bonn and Nijmegen NN interactions $V_{N N}$. By construction, the high momentum modes of the original $V_{N N}$ are integrated out in $V_{\text {low-k }}$, with the requirement that the deuteron binding energy and low energy phase shifts of $V_{N N}$ are exactly reproduced. Using this interaction, we evaluate the bulk properties (binding energy and saturation density) of nuclear matter and finite nuclei, in particular their dependence on the cut-off parameter. We also study the pairing gap and the residual interaction in nuclear matter in terms of the Landau parametrization. At low and medium densities, the HF and BHF binding energies for nuclear matter calculated with the $V_{\text {low-k }}$ 's derived from the CD-Bonn and Nijmegen potentials are nearly identical. The pairing gaps and Landau parameters derived from $V_{\text {low-k }}$ are remarkably close to those given by the full-space $V_{N N}$. The $V_{\text {low-k }}$ interactions, however, fail to reproduce the saturation property of nuclear matter at higher densities if the cut-off for the high momentum modes is assumed density independent.
\end{abstract}

PACS: 21.30.Fe; 13.75.Cs; 27.80.+j; 21.60.-n;

Keywords: Forces in hadronic systems and effective interactions; Nucleon-Nucleon Interactions; Properties of nuclei; Nuclear-structure: models and methods

\section{Introduction}

In the past decade, there has been much progress in treating low-energy nuclear systems using the effective field theory (EFT) and renormalization group (RG) approaches, $[1,2,3,4,5,6,7,8]$ and references therein. The fundamental theory of strong interaction, QCD, is non-perturbative in the energy regime characteristic for low energy nuclear physics. This poses a major difficulty for a proper derivation of the nuclear force from the first principles. However, this 
difficulty can be overcome by the EFT-RG approach where one "integrates out" the fast fields beyond the chiral symmetry breaking scale $\Lambda_{\chi} \approx 1 \mathrm{GeV}$. Well below this scale, the appropriate degrees of freedom for nuclear systems are pions and nucleons. In this way, one obtains for these degrees of freedom a chiral symmetric EFT, which is used to construct either perturbative or non-perturbative controlled approximations for low energy nuclear systems.

As is well known, there are several realistic models for the NN potential $V_{N N}$, such as the CD-Bonn [9], Nijmegen [10] and the Argonne [11] potentials. These models all have the same long range tail, namely the one-pion exchange potential. But the short range parts significantly differ from each other, despite the fact they all fit essentially exactly the empirical deuteron binding energy and low energy phase shifts up to $E_{l a b} \approx 350 \mathrm{MeV}$. Clearly the short range (high momentum) parts of these potentials are model dependent and are different e.g. for the local and non-local interaction models. Employing the EFT-RG ideas, Refs. [12,13,14] "integrated out" the high momentum modes of these potentials and thereby obtained a low momentum NN potential $V_{\text {low-k }}$. The high momentum modes of the original $V_{N N}$ are eliminated in $V_{\text {low-k }}$ with the requirement that the deuteron binding energy and low energy phase shifts of $V_{N N}$ are exactly reproduced. This is in line with the central theme of the EFT$\mathrm{RG}$ approach that physics in the infrared region is insensitive to the details of the short distance dynamics.

The purpose of this work is to calculate various nuclear structure observables for infinite nuclear matter and finite nuclei starting from the $V_{\text {low-k }}$ interaction derived for the CD-Bonn and Nijmegen potentials [12,13,14]. In particular, we would like to explore to which extent the results derived from $V_{\text {low-k }}$ differ from the predictions obtained for the underlying interaction model $V_{N N}$ and the sensitivity of the results on the cut-off parameter $\Lambda$. For that purpose we evaluate the ground-state energy of nuclear matter and finite nuclei taking ${ }^{16} \mathrm{O}$ as a typical example. Apart from the bulk properties of nuclear systems we also investigate the predictions for the residual interaction characterized by the pairing gap $\Delta$ of neutron-neutron or proton-proton pairing as well as the Landau parametrization of the particle-hole interaction for low-energy excitations in nuclear matter.

The paper is organized as follows. In section 2 we shortly review the construction of the low-momentum $V_{\text {low }-\mathrm{k}}$. The methods for the evaluation of ground-state properties of nuclear matter and finite nuclei as well as the corresponding results are presented in sections 3 and 4, respectively. Section 5 contains a discussion of the gap equation and the Landau parameters characterizing the residual interaction. The conclusions of this study are summarized in section 6 . 


\section{Determination of low momentum nucleon-nucleon potentials}

We start with a brief review of the method of carrying out the high-momentum decimation $[12,13,14]$. In accordance with the general definition of a renormalization group transformation, the decimation must be such that low energy observables calculated from the full $V_{N N}$ must be reproduced by $V_{\text {low-k }}$, but with all loop integrals cut off at some $\Lambda$. To meet this requirement, a $T$-matrix equivalence approach is used $[12,13,14]$. One starts from the half-on-shell $T$ matrix

$$
T\left(k^{\prime}, k, k^{2}\right)=V_{N N}\left(k^{\prime}, k\right)+\int_{0}^{\infty} q^{2} d q V_{N N}\left(k^{\prime}, q\right) \frac{m}{k^{2}-q^{2}+i 0^{+}} T\left(q, k, k^{2}\right),
$$

and then defines an effective low-momentum $T$-matrix by

$T_{\text {low-k }}\left(p^{\prime}, p, p^{2}\right)=V_{\mathrm{low}-\mathrm{k}}\left(p^{\prime}, p\right)+\int_{0}^{\Lambda} q^{2} d q V_{\mathrm{low}-\mathrm{k}}\left(p^{\prime}, q\right) \frac{m}{p^{2}-q^{2}+i 0^{+}} T_{\mathrm{low}-\mathrm{k}}\left(q, p, p^{2}\right)$,

where $\Lambda$ denotes the momentum space cut-off. The above $T$-matrices satisfy the condition

$$
T\left(p^{\prime}, p, p^{2}\right)=T_{\mathrm{low}-\mathrm{k}}\left(p^{\prime}, p, p^{2}\right) ; \quad \text { for } \quad\left(p^{\prime}, p\right) \leq \Lambda .
$$

$V_{\text {low-k }}$ has been obtained by solving the above equations using the procedures of Ref. $[12,13]$. In particular the effects of the folded diagrams are taken into account to derive an effective interaction $V_{\text {low-k }}$ which is energy-independent.

We have performed numerical checks to make sure that the resulting $V_{\text {low-k }}$ does preserve the deuteron binding energy and low energy phase shifts of the original NN potential. This has been repeated assuming various cut off parameters $\Lambda$ using the CD Bonn [9] and the Nijmegen [10] interaction models for the bare interaction $V_{N N}$. For both of these interactions we have ignored the charge dependence and used only the proton-neutron part for all interactions channels.

\section{Energy of Nuclear Matter}

It is well known that the strong short-range and tensor components of realistic NN interactions induce strong $\mathrm{NN}$ correlations in the wave function of nuclear many-body system. Therefore rather sophisticated approximation schemes for the solution of the many-body problem are required to obtain reasonable results for nuclear structure observables. Such approximation schemes must account at least for the two-nucleon correlations. Typical examples are variational approaches using correlated basis functions [15], the coupled cluster 
approach [16], the self-consistent evaluation of Green's functions [17] and the hole-line expansion within the Brueckner-Hartree-Fock (BHF) approximation [18].

The effective interaction $V_{\text {low-k }}$ has been constructed to account for NN correlations with momenta larger than the cut-off momentum $\Lambda$ as they occur for two interacting nucleons in the vacuum. The question which we now want to address is whether such a treatment of correlations is sufficient to allow for simple mean field or Hartree-Fock calculations of the nuclear many-body system. As a first example we consider the equation of state of infinite nuclear matter.

The left panel of Fig. 1 shows the results of Hartree-Fock (HF) calculations for the energy per nucleon of symmetric nuclear matter as a function of density, which is characterized in this figure by the corresponding Fermi momentum $k_{F}$. For the bare CD-Bonn interaction [9] the HF calculations yield positive energies ranging between $3 \mathrm{MeV}$ per nucleon at small densities and $20 \mathrm{MeV}$ per nucleon for $k_{F}$ around $1.8 \mathrm{fm}^{-1}$ which corresponds to a density of around 2.3 times the empirical saturation density. This demonstrates the well known fact that even modern non-local models for a realistic NN interaction, which tend to be "soft", yield unbound nuclei if the effects of correlations are ignored [19].

This lack of binding energy can be cured if the effects of NN correlations are taken into account, e.g. by means of the BHF approximation. In the BHF approximation one replaces the bare interaction by the $G$-matrix which obeys the Bethe-Goldstone equation. For a pair of nucleons with vanishing center of mass momentum it takes the form

$$
G\left(k^{\prime}, k, 2 \varepsilon_{k}\right)=V_{N N}\left(k^{\prime}, k\right)+\int_{0}^{\infty} q^{2} d q V_{N N}\left(k^{\prime}, q\right) \frac{\hat{Q}}{2 \varepsilon_{k}-2 \varepsilon_{q}+i 0^{+}} G\left(q, k, 2 \varepsilon_{k}\right) .
$$

Comparing this equation with the Lippmann-Schwinger equation (1), one can see that the $G$-matrix is closely connected to the $T$-matrix except for the fact that the Pauli operator $\hat{Q}$ in (4) prevents scattering into states which are occupied by other nucleons and that the propagator for the two nucleons, which for the vacuum is defined in terms of the kinetic energies (see eq. (1)) is replaced in (4) by the propagator in the medium defined in terms of the singleparticle energies $\varepsilon_{k}$. Both of these modifications tend to reduce the effects of correlations and are referred to as Pauli and dispersive quenching, respectively $[20]$.

It should be mentioned that we employ a very simple version of the BHF approach, in which the single-particle energies for states below the Fermi energy 
are parametrized in terms of an effective mass and a constant energy shift

$$
\varepsilon_{k}=\frac{k^{2}}{2 m^{*}}+U
$$

while for states above the Fermi energy the single-particle energies are replaced by the kinetic energy (the so-called conventional choice). The Pauli operator is treated in the angle-averaged approximation. A more sophisticated treatment of the two-nucleon propagator in the Bethe-Goldstone equation leads to larger binding energies [21]. Note, however, that it is not the aim of this study to optimize the BHF approximation, but to study the possible use of the $V_{\text {low-k }}$ interaction in restricted model-space calculations.

Results of such BHF calculations are displayed in the right panel of Fig. 1. The conventional BHF calculation for the bare CD Bonn interaction yields an energy of $-17 \mathrm{MeV}$ per nucleon and a saturation density around twice the empirical saturation density. The left panel of Fig. 1 also shows the HF energies calculated for $V_{\text {low-k }}$ assuming three different values for the cut-off parameter $\Lambda$. The resulting energies are attractive. This demonstrates that the correlations from two-particle states with momenta $q$ larger than the cut-off $\Lambda$, which are accounted for in $V_{\text {low-k }}$, are sufficient to provide this attraction. From this consideration it is evident that the energies are more attractive the smaller the cut-off $\Lambda$, a feature seen in Fig. 1.

Next, in performing BHF calculations for $V_{\text {low-k }}$, we must take into account that $V_{\text {low-k }}$ is designed for a model space with relative momenta smaller than $\Lambda$, i.e. the matrix elements of $V_{\text {low-k }}$ must be supplemented by the value zero for momenta $q$ larger than the cut-off. In other words, the integral in the Bethe-Goldstone eq. (4) is restricted to momenta $q$ below the cut-off in order to avoid double counting of the contributions from momenta higher than the cut-off.

The energies resulting from such BHF calculations (see left panel of Fig. 1) are below the corresponding $\mathrm{HF}$ energies as additional correlations from NN states above the Fermi momentum but below the cut-off are taken into account. One also observes that the BHF calculations using $V_{\text {low-k }}$ lead to more attractive energies than the BHF calculation using the bare interaction. This is due to the fact, that the dispersive quenching effects in the $G$-matrix discussed above are taken into account only for intermediate two-particle states with momenta below the cut-off $\Lambda$, while the BHF calculation using the bare interaction considers these effects for all states. Since the dispersive quenching is a very important ingredient of BHF calculations, that provides the saturation effect, the BHF calculations employing $V_{\text {low-k }}$ do not exhibit a minimum in the energy versus density curve. One may conclude that $V_{\text {low-k }}$ yields a good approximation for BHF calculations at small densities. In this regime even a Hartree-Fock calculation leads to reasonable results, if $\Lambda$ is small enough. At 
higher densities, however, $V_{\text {low-k }}$ leads to too much binding as it does not account for the dispersive quenching of high-momentum modes.

In Fig. 2 we compare results derived from two different NN interaction models: the CD-Bonn (used above) and the Nijmegen [10] potentials. One can see from this figure that the HF calculations using the Nijmegen interaction yield energies which are more repulsive than those derived from the CD-Bonn interaction. The non-local CD-Bonn is "softer" than the local Nijmegen interaction. As it has been discussed e.g. in Ref. [19] the softer interaction model yields more attractive energies also within the BHF approximation (see right panel of Fig. 2). Although the results of $\mathrm{HF}$ as well as BHF calculations are rather sensitive to the choice of the interaction, the corresponding results from $V_{\text {low-k }}$ are rather insensitive to the interaction model on which they are based. This is true for both the HF and the BHF approximations. This can be understood from the fact that the $T$-matrix is essentially independent on the interaction used, as all realistic interactions are fitted to the empirical phase shifts. Since the definition of $V_{\text {low-k }}$ is related to the $T$-matrix (see eq. (3)), the resulting effective interaction is rather insensitive to the original interaction it is based on.

\section{Finite Nuclei}

Since the use of $V_{\text {low-k }}$ yields rather reasonable results for the energy of nuclear matter at small densities it is of interest to investigate the predictions of $V_{\text {low-k }}$ for the ground-state properties of light nuclei. For that purpose we employ the calculation scheme, which has recently been discussed in ref. [22] and apply it for the nucleus ${ }^{16} \mathrm{O}$. This scheme is an extension of the conventional BHF approximation for finite nuclei. It assumes a model space, which in our example is defined in terms of configurations of nucleons occupying oscillator states up to the $1 p 0 f$ shell. Correlations outside this model space, which we would like to call short-range correlations, are treated within the framework of the BHF approximation. The long-range correlations from configurations inside the model space are treated within the framework of the Green's function approach, assuming a self-energy which accounts for 2-particle - 1-hole and 2-hole - 1-particle admixtures.

This Green's function approach (for further details see ref. [22]) yields the spectral function of the hole-state distribution $S_{\tau l j}^{h}(\omega)$, which is the probability to remove a nucleon with quantum numbers $\tau$ for isospin, $l$ for orbital angular momentum and $j$ for the total angular momentum by absorption of an energy $-\omega$. From this spectral function we can easily determine the total occupation 
probability

$$
\rho_{\tau l j}=\int_{-\infty}^{\varepsilon_{F}} d \omega S_{\tau l j}^{h}(\omega)
$$

the mean single-particle energy of the distribution function

$$
\varepsilon_{\tau l j}=\int_{-\infty}^{\varepsilon_{F}} d \omega \omega S_{\tau l j}^{h}(\omega)
$$

and the total energy

$$
E=\sum_{\tau l j} \frac{(2 j+1)}{2} \int_{-\infty}^{\varepsilon_{F}} d \omega S_{\tau l j}^{h}\left(\omega_{\tau l j}\right)\left\langle\frac{p^{2}}{2 m}+\omega_{\tau l j}\right\rangle .
$$

Results for these observables are listed in table 1 using the bare CD-Bonn interaction along with $V_{\text {low-k }}$ derived from the CD-Bonn potential with $\Lambda=2$ $\mathrm{fm}^{-1}$ and $3 \mathrm{fm}^{-1}$. For the interactions $V_{\text {low }-\mathrm{k}}$ we have performed calculations in which the effects of short-range correlations are ignored (labeled HF) and taken into account (labeled BHF).

The BHF calculations employing $V_{\text {low-k }}$ yield results which are very close to the corresponding ones for the bare interaction. The agreement is better for the larger value of the cut-off $\Lambda$. The BHF calculations using $V_{\text {low-k }}$ yield energies which are more attractive than the corresponding result for the bare interaction. This can be explained in the same way as for the case of nuclear matter: $V_{\text {low-k }}$ accounts for correlations originating from states above the cutoff as they occur for two free nucleons. The dispersive quenching effect in the $G$-matrix calculated for the bare interaction, however, reduces these attractive correlation contributions. We find that this effect is rather small for finite nuclei, comparable to the same effect in nuclear matter at small densities (see Fig. 1).

The predictions from calculations using $V_{\text {low-k }}$ in the HF approach yield energies which are less attractive than those from the bare interaction. This is due to the fact that they do not account for short-range correlations originating from configurations outside the model space (up to $1 p 0 f$ shell) but only below the cut-off. Nevertheless, the results obtained for $\Lambda=2 \mathrm{fm}^{-1}$ are quite reasonable.

The predictions for the occupation probabilities are larger for the calculations using $V_{\text {low-k }}$ than for the bare interaction $V$. This is due to the fact that the HF calculations using $V_{\text {low-k }}$ only account for a depletion of the occupation due to long-range correlations within the $1 p 0 f$ model space, while the corresponding BHF calculations include effects of correlations only up to the cut-off $\Lambda$. One may observe, however, that the BHF calculation using $V_{\text {low-k }}$ with $\Lambda=3 \mathrm{fm}^{-1}$ yields results for the occupation which are only slightly above those derived from the bare interaction. 
The reliability of $V_{\text {low-k }}$ in low-energy nuclear structure calculations of finite nuclei is also supported by the results for the spectral function displayed in Fig. 3. The structure of the spectral function derived from the bare interaction, represented by the dashed line in both parts of the figure, is nicely reproduced by the spectral function derived from $V_{\text {low-k }}$. This is true in particular for the BHF approach, displayed by the solid line in the upper panel, but also holds for the HF approximation, which is shown in the lower panel. The main deficiency is a shift of the strength to larger energies $\omega$, a feature which we have already outlined above in discussing the mean single-particle energies shown in table 1.

\section{$5 \quad$ Pairing and Residual Interaction in Nuclear Matter}

Up to this point we have focused the discussion on observables, which are characteristic for the ground state of nuclear systems. In this section we would like to address the question how reliable is the use of $V_{\text {low-k }}$ for observables related to the low-energy excitation spectrum. As the first example we consider the evaluation of the pairing gap in nuclear matter. In particular we take the example of neutron-neutron pairing in the ${ }^{1} S_{0}$ channel for neutron matter at $k_{F}=1.36 \mathrm{fm}^{-1}$. This pairing gap is determined from the solution of the gap equation (see e.g. [23,24])

$$
\Delta(k)=-\frac{2}{\pi} \int_{0}^{\infty} d k^{\prime} k^{\prime 2} V\left(k, k^{\prime}\right) \frac{\Delta\left(k^{\prime}\right)}{2 \sqrt{\left(\varepsilon_{k}^{\prime}-\varepsilon_{F}\right)^{2}+\Delta\left(k^{\prime}\right)^{2}}} .
$$

Here we will again parametrize the single-particle spectrum by eq. (5) and consider the cases $m^{*}=m$ and $m^{*}=700 \mathrm{MeV}$. For a realistic interaction, one has to integrate up to rather large values of $k^{\prime}$ before a convergent result is obtained. Corresponding results for $\Delta(k)$ for the bare CD-Bonn interaction

are displayed in Fig. 4 . For the $V_{\text {low-k }}$ interactions, the integral in (9) can be constrained to values of $k^{\prime}$ below the cut-off $\Lambda$.

The agreement for the resulting gap functions is very good for momenta below the cut-off $\Lambda$ and a single-particle spectrum with an effective mass identical to the bare mass. In this case the use of $V_{\text {low }-\mathrm{k}}$ in the gap equation corresponds to the use of the effective model-space interaction of ref. [24]. However, if we use a single-particle spectrum renormalized by the medium, the results obtained from $V_{\text {low-k }}$ differ in a more significant way from the corresponding result obtained for the bare CD-Bonn interaction. If, e.g. we use an effective mass $m^{*}$ of $700 \mathrm{MeV}$ (see right panel of Fig. 4), $V_{\text {low-k }}$ tends to overestimate the value for the gap $\Delta$ as it does not account for the reduction of the contributions from high lying NN states, which is due to the decrease of the effective mass. 
This feature is identical to the effect of the dispersive quenching of correlations discussed above.

Finally, we would like to consider the residual interaction for low-energy excitations as characterized by the Landau parameters. It is not our goal to employ a very sophisticated calculational scheme for the Landau parameters. This would involve in particular a careful analysis of the contributions due to the induced interaction terms $[25,26,27]$. Our aim here is to compare the results from the bare interaction $V_{N N}$, the corresponding $G$-matrix and those derived from $V_{\text {low-k }}$, in order to see to which extent $V_{\text {low-k }}$ accounts for the short-range correlation effects in a nuclear system. For that purpose, we determine the Landau parameters either from the bare interaction or the $G$-matrix [28].

The results of our calculations of the Landau parameters are listed in table 2. Beside those parameters we also list the effective mass determined by

$$
\frac{m^{*}}{m}=1+\frac{1}{3} f_{1}
$$

and the coefficient $\beta$ for the symmetry energy

$$
\beta=\frac{1}{3} \frac{k_{F}^{2}}{2 m^{*}}\left(1+f_{0}^{\prime}\right) .
$$

Comparing the various approximations with our "best" result derived from the $G$-matrix of the CD-Bonn potential, one finds that the bare interaction $V_{\text {low-k }}$ with a small cut-off parameter yields a good approximation if one would like to restrict oneself to the bare interaction. If an iteration is carried out in the particle-particle channels for momenta up to the cut-off $\Lambda$, one obtains the best results using a larger cut-off.

\section{Conclusions}

The aim of this study was to explore to which extent the effects of shortrange correlations, which are due to excited states above a cut-off parameter $\Lambda$, are accounted for by the effective interaction $V_{\text {low }-\mathrm{k}}$, which has recently been constructed $[12,13,14]$. This effective interaction accounts for short-range correlations of two-nucleons interacting in the vacuum; the question is whether this is a good approximation also for the corresponding correlations of nucleons interacting in a nuclear medium. To study this question we considered various nuclear structure observables.

Inspecting the equation of state calculated for nuclear matter one may conclude that $V_{\text {low-k }}$ yields a good approximation for BHF calculations at small 
densities. In this regime even a Hartree-Fock calculation leads to reasonable results, if $\Lambda$ is small enough. At higher densities, however, $V_{\text {low-k }}$ produces too much binding as it does not account for the dispersive quenching effects, which are taken into account e.g. in the $G$-matrix. Therefore calculations using $V_{\text {low-k }}$ cannot reproduce the saturation feature of nuclear matter.

The $V_{\text {low-k }}$ interaction is well suited for studies of finite nuclei like ${ }^{16} \mathrm{O}$, which is again a system of small density. In this case we obtain reasonable agreement for the calculated energies, but also for the spectral function for nucleon removal.

Furthermore, we demonstrated that the use of $V_{\text {low-k }}$ is also very efficient in calculating quantities like the pairing gap in infinite nuclear matter. The agreement with the exact result is very good if one may replace the single-particle energy above the cut-off by pure kinetic energy. Deviations are observed if this is not the case. These deviations are again related to the dispersive quenching mechanism mentioned above.

In conclusion we would like to emphasize that $V_{\text {low-k }}$ can be a very useful tool for low-energy nuclear structure calculations. However, one should be cautious if the observable of interest is sensitive to the single-particle spectrum at energies above the cut-off. This is a problem in particular for high densities.

\section{Acknowledgements}

We thank Prof. T.T.S. Kuo for supplying the matrix elements of $V_{\text {low-k }}$ and for many stimulating discussions. This work was supported in part by the DFG (SFB 382) and through a stipend of the DAAD.

\section{References}

[1] S. Weinberg, Phys. Lett. B251 (1990) 288; Nucl. Phys. B363 (1991) 3.

[2] D.B. Kaplan, M.J. Savage and M.B. Wise, Phys. Lett. B424 (1998) 390; Nucl. Phys. B534 (1998) 329.

[3] P. Lepage, "How to Renormalize the Schroedinger Equation", (1997) nucth/9706029

[4] R. Shankar, Rev. Mod. Phys. 66 (1994) 129.

[5] P. Bedaque et. al. (eds.), Nuclear Physics with Effective Field Theory II, (1999) World Scientific Press.

[6] U. van Kolck, Prog. Part. Nucl. Phys. 43 (1999) 337. 
[7] E. Epelbaum, W. Glöckle, and Ulf-G. Meissner, Nucl. Phys. A637 (1998) 107; ibid. A671 (2000) 295.

[8] W. Haxton and C.L. Song, Phys. Rev. Lett. 84 (2000) 5484.

[9] R. Machleidt, Phys. Rev. C63 (2001) 024001.

[10] V.G.J. Stoks, R.A.M. Klomp, C.P.F. Terheggen, and J.J. de Swart, Phys. Rev. C49 (1994) 2950.

[11] R.B. Wiringa, V.G.J. Stoks, and R. Schiavilla, Phys. Rev. C51 (1995) 38.

[12] S. K. Bogner, T.T.S. Kuo and L. Coraggio, Nucl. Phys. A684 (2001) 432c.

[13] S. K. Bogner, T.T.S. Kuo, L. Coraggio, A. Covello and N. Itaco, Phys. Rev. C65 (2002) 05130(R).

[14] S. K. Bogner, A. Schwenk, T.T.S. Kuo and G.E. Brown, Nucl. Phys. A (2002)(to be published).

[15] A. Akmal and V.R. Pandharipande, Phys. Rev. C56 (1997) 2261.

[16] H. Kümmel, K.H. Lührmann, and J.G. Zabolitzky, Phys. Rep. 36 (1978) 1.

[17] W.H. Dickhoff and H. Müther, Reports on Prog. in Phys. 11 (1992) 1947.

[18] H. Müther and A. Polls, Prog. Part. and Nucl. Phys. 45 (2000) 243.

[19] H. Müther and A. Polls, Phys. Rev. C61 (2000) 014304.

[20] R, Machleidt, Adv. in Nucl. Phys. 19 (1989) 189.

[21] T. Frick, Kh. Gad, H. Müther, and P. Czerski, Phys. Rev. C65 (2002) 034321.

[22] Kh. Gad and H. Müther, Phys. Rev. C, in print, preprint nucl-th/0205025.

[23] M. Baldo, J. Cugnon, A. Lejeune, and U. Lombardo, Nucl. Phys. A515 (1990) 409.

[24] Ø. Elgarøy , L. Engvik, M. Hjorth-Jensen, and E. Osnes, Nucl. Phys. A604 (1996) 466

[25] S. Babu and G.E. Brown, Ann. of Phys. 78 (1973) 1.

[26] W.H. Dickhoff, A. Faessler, H. Müther, and S.S. Wu, Nucl. Phys. A405 (1983) 534.

[27] A. Schwenk, B. Friman, and G.E. Brown, preprint, nucl-th/0207004.

[28] W.H. Dickhoff, A. Faessler, J. Meyer-ter-Vehn, and H. Müther, Nucl. Phys. A368 (1981) 445. 


\begin{tabular}{|c|c|c|c|c|c|c|}
\hline & \multicolumn{3}{|c|}{$\mathrm{HF}$} & \multicolumn{3}{|l|}{$\mathrm{BHF}$} \\
\hline & $\Lambda=2 \mathrm{fm}^{-1}$ & $\Lambda=3 \mathrm{fm}^{-1}$ & $\Lambda=2 \mathrm{fm}^{-1}$ & $\Lambda=3 \mathrm{fm}^{-1}$ & bare $\mathrm{V}$ & Exp \\
\hline$\varepsilon_{s 1 / 2}$ & -39.28 & -31.31 & -45.23 & -44.74 & -44.00 & $-40 \pm 8$ \\
\hline$\varepsilon_{p_{3} / 2}$ & -18.32 & -12.34 & -25.07 & -24.83 & -24.29 & -18.45 \\
\hline$\varepsilon_{p_{1} / 2}$ & -15.01 & -9.01 & -21.97 & -21.81 & -21.26 & -12.13 \\
\hline$\rho_{s 1 / 2}$ & 0.964 & 0.964 & 0.906 & 0.880 & 0.867 & \\
\hline$\rho_{p 3 / 2}$ & 0.928 & 0.936 & 0.844 & 0.828 & 0.823 & \\
\hline$\rho_{p 1 / 2}$ & 0.912 & 0.912 & 0.832 & 0.815 & 0.802 & \\
\hline $\mathrm{E} / \mathrm{A}$ & -4.96 & -1.69 & -8.22 & -8.08 & -7.78 & -7.98 \\
\hline
\end{tabular}

Table 1

Single-particle energies $\left(\varepsilon_{i}\right)$ and occupation probabilities $\left(\rho_{i}\right)$ for protons in ${ }^{16} \mathrm{O}$ and the total energy per nucleon (E/A) as calculated from various approximations and interactions are compared to the experimental data. The CD Bonn interaction has been used (bare $\mathrm{V}$ ) and the effective interactions $V_{\mathrm{low}-\mathrm{k}}$ derived from it assuming a cut-off $\Lambda$ of $2.0 \mathrm{fm}^{-1}$ and $3.0 \mathrm{fm}^{-1}$. The effects of short-range correlations have been ignored (HF) or taken into account by means of the BHF approximation. All energies are given in $\mathrm{MeV}$.

\begin{tabular}{c|rrr|rrr} 
& \multicolumn{6}{|c}{$V$} \\
& $\Lambda=2 \mathrm{fm}^{-1}$ & $\Lambda=3 \mathrm{fm}^{-1}$ & bare $\mathrm{V}$ & $\Lambda=2 \mathrm{fm}^{-1}$ & $\Lambda=3 \mathrm{fm}^{-1}$ & bare V \\
\hline$f_{0}$ & -1.819 & -1.315 & -0.465 & -2.003 & -1.807 & -1.601 \\
$f_{0}^{\prime}$ & 0.502 & 0.235 & 0.072 & 0.614 & 0.492 & 0.422 \\
$g_{0}$ & 0.250 & 0.362 & 0.226 & 0.211 & 0.279 & 0.276 \\
$g_{0}^{\prime}$ & 0.970 & 0.899 & 0.709 & 0.998 & 0.973 & 0.929 \\
$h_{0}$ & 1.788 & 1.759 & 1.618 & 1.753 & 1.717 & 1.694 \\
$h_{0}^{\prime}$ & -0.596 & -0.631 & -.581 & -.588 & -.620 & -.613 \\
\hline$m^{*} / m$ & 0.702 & 0.684 & 0.620 & 0.696 & 0.688 & 0.676 \\
$\beta[\mathrm{MeV}]$ & 28.98 & 24.46 & 23.43 & 31.40 & 29.35 & 28.48
\end{tabular}

Table 2

Landau parameters, effective mass and the coefficient for the symmetry energy $\beta$ as calculated from the interaction $V$ and the corresponding $G$ matrix in nuclear matter at a Fermi momentum $k_{F}$ of $1.36 \mathrm{fm}^{-1}$. The CD Bonn interaction has been used (bare $\mathrm{V}$ ) and the effective interactions $V_{\text {low-k }}$ derived from it assuming a cut-off $\Lambda$ of $2.0 \mathrm{fm}^{-1}$ and $3.0 \mathrm{fm}^{-1}$. The Landau parameters are normalized with respect to the density of states at the Fermi momentum using an effective mass as listed. 


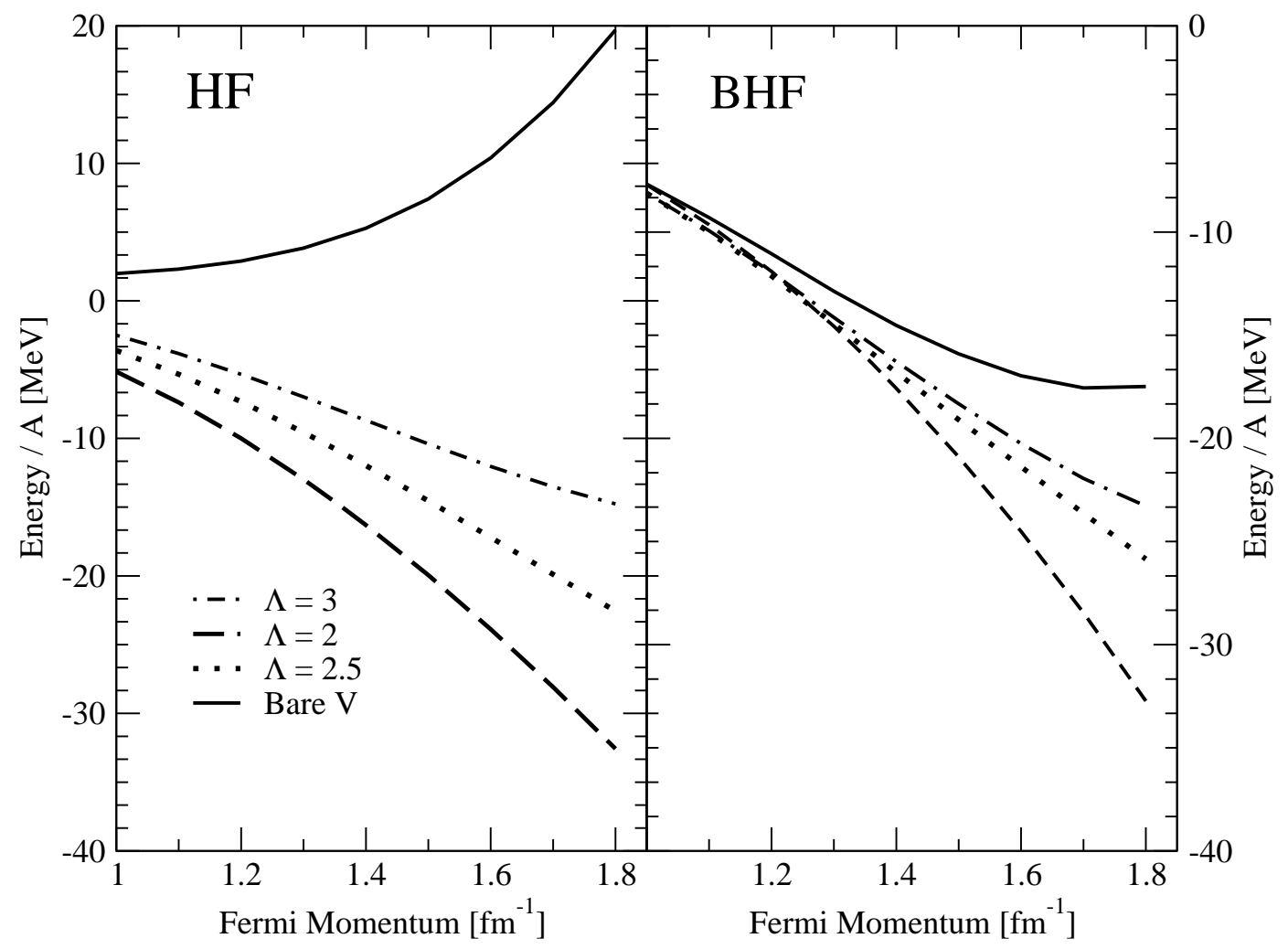

Fig. 1. Binding energy of nuclear matter as a function of the Fermi momentum for the HF (left panel) and BHF approximations (right panel). The bare interaction (CD-Bonn [9]) and corresponding $V_{\text {low-k }}$ interactions with various cut-off parameters $\Lambda$ were used. 


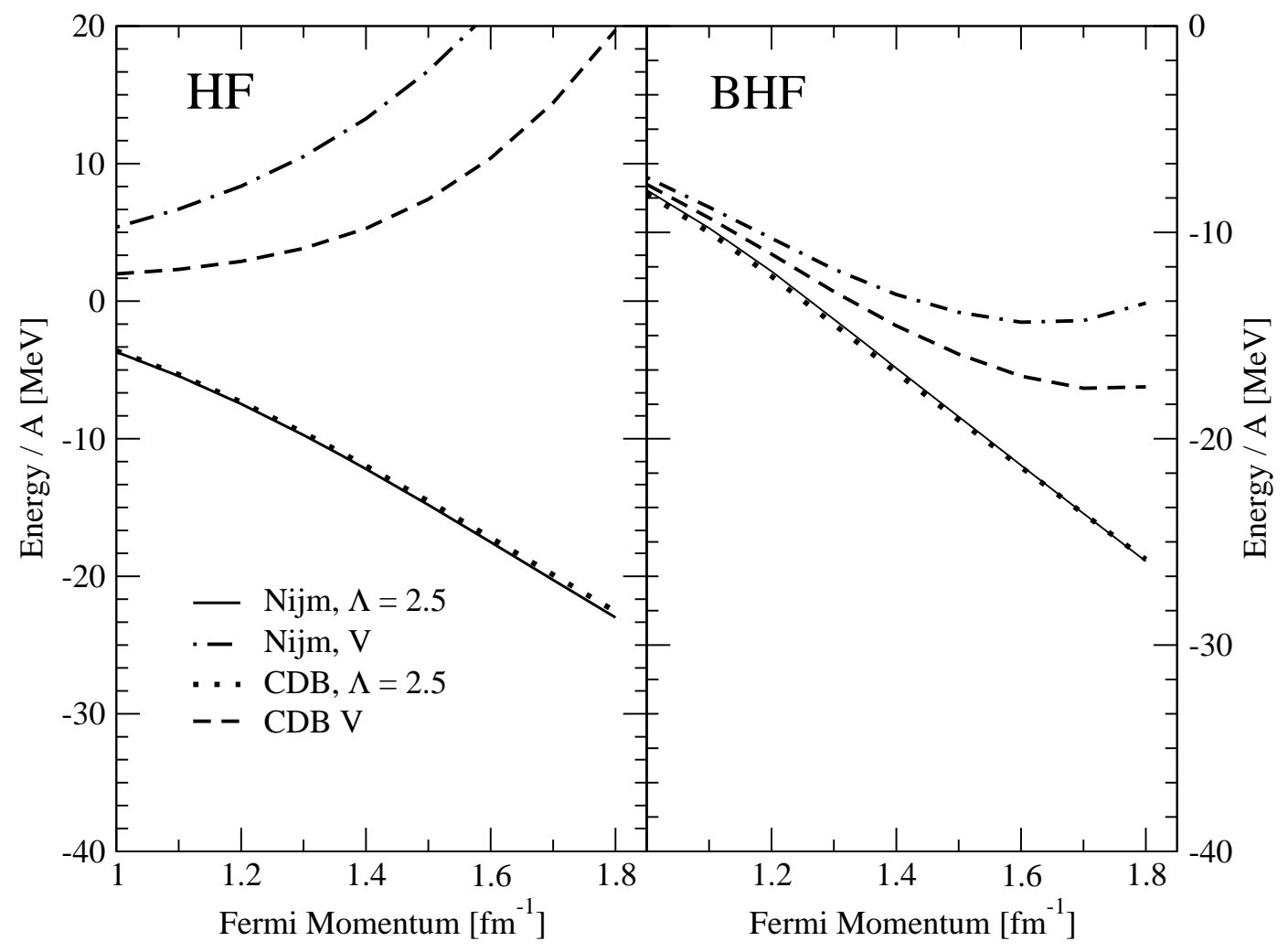

Fig. 2. Binding energy of nuclear matter as a function of the Fermi momentum for the HF (left panel) and BHF approximations (right panel). The interactions are modeled by the CD-Bonn and Nijmegen potentials and corresponding $V_{\text {low-k }}$ interactions with a cut-off parameter $\Lambda=2.5 \mathrm{fm}^{-1}$. 


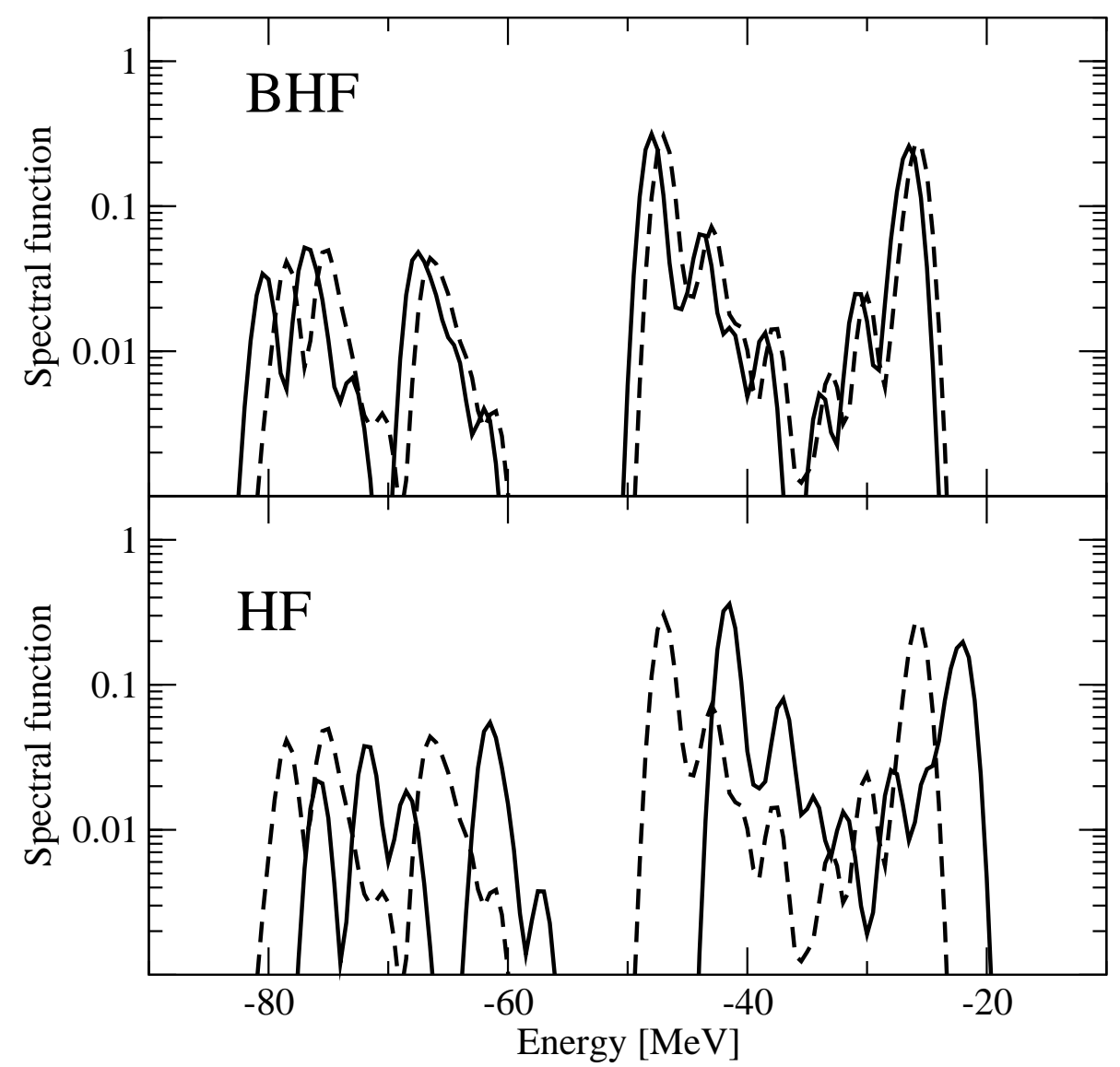

Fig. 3. The spectral function for proton hole strength in ${ }^{16} \mathrm{O}$ in the $s_{1 / 2}$ channel for the BHF (upper panel) and the HF (lower panel) approximations. The spectral distribution shown by the solid line has been calculated for $V_{\text {low-k }}$ with $\Lambda=2 \mathrm{fm}^{-1}$. For comparison, we show the results derived from the BHF approximation using the bare interaction (dashed lines)[22]. 


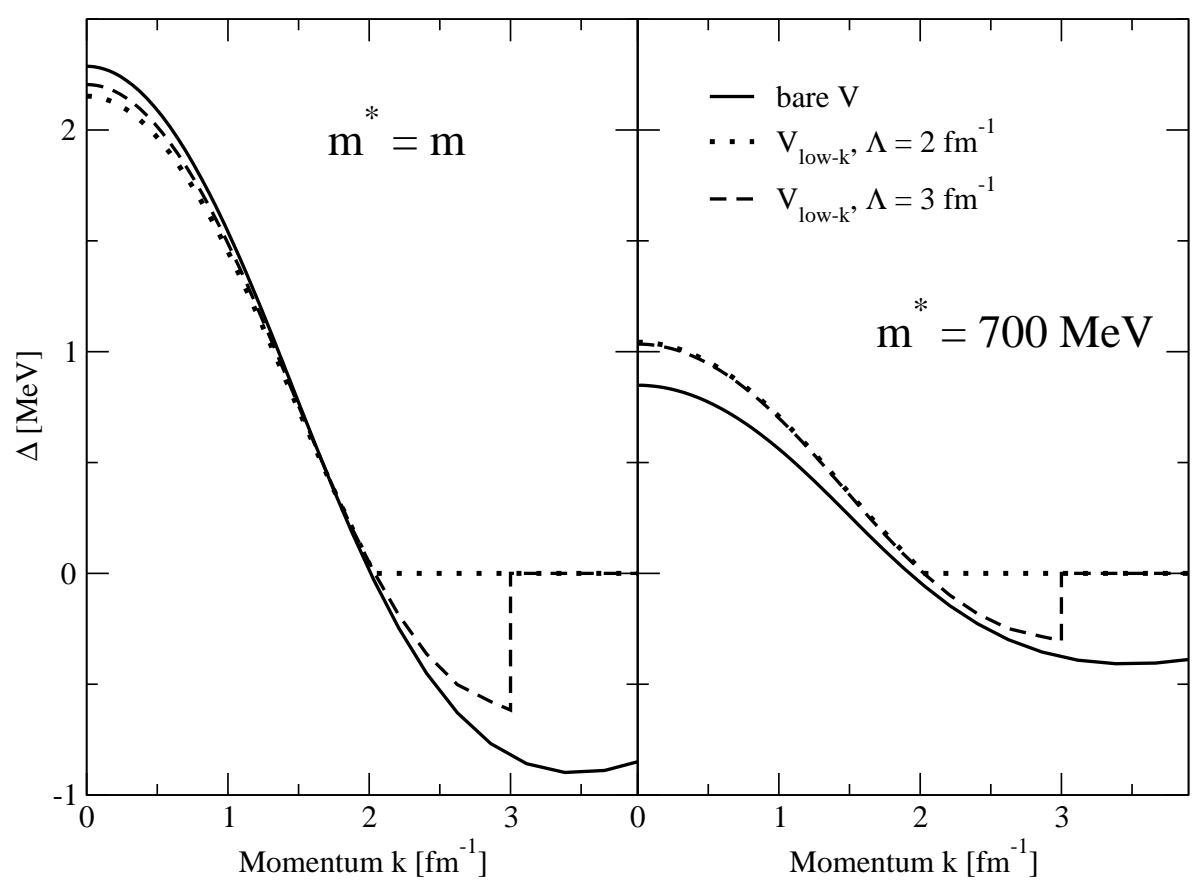

Fig. 4. The pairing gap $\Delta(k)$ for neutron-neutron pairing in the ${ }^{1} S_{0}$ channel for neutron matter with a Fermi momentum $k_{F}=1.36 \mathrm{fm}^{-1}$ as function of the nucleon momentum $k$. The results obtained for the bare CD-Bonn interaction (solid lines) are compared to those derived from $V_{\text {low-k }}$ using cut-offs $\Lambda=2 \mathrm{fm}^{-1}$ and $3 \mathrm{fm}^{-1}$. The left and right panels show the results for non-renormalized single-particle spectrum $\left(m^{*}=m\right)$ and for the renormalized spectrum with an effective mass $m^{*}=700 \mathrm{MeV}$, respectively. 